\title{
IMPLICATIONS OF WHITE-DWARF CRYSTALLIZATION FOR THE CHEMICAL COMPOSITION OF THE PLANETARY NUCLEI
}

\author{
H. M. VAN HORN \\ (Dept. of Physics and Astronomy, University of Rochester, U.S.A.)
}

It now seems to be reasonably well-established that the central stars of planetary nebulae evolve directly into white dwarfs. Evidently a knowledge of the chemical composition of the white dwarfs would therefore be of considerable importance in helping to identify the point in the evolution at which the mechanism responsible for expulsion of the nebular shell becomes operative. For this reason I would like to present some evidence which provides a direct suggestion for the internal composition of some of the white dwarfs and to examine briefly the implications of this suggestion for the relation between the planetary nuclei and the white dwarfs.

It was first pointed out by Kirzhnits (1960) and independently by Salpeter (1961) that matter in the interior of a cold 'white' dwarf should be in a crystalline rather than a gaseous state. More recently Mestel and Ruderman (1967) have shown that crystallization will begin in a white dwarf of central density $\rho$ when the central temperature $T$ falls to a value of

$$
T \sim 3 \times 10^{5}\left(\frac{\rho}{10^{6}}\right)^{1 / 3} Z^{5 / 3}{ }^{\circ} \mathrm{K}
$$

where $\rho$ is in $\mathrm{g} \mathrm{cm}^{-3}$, and $Z$ is the mean nuclear charge. In this phase transition there is a release of energy in the form of the heat of crystallization, $\Delta Q \sim k T$, where $T$ is the crystallizing temperature. The release of this energy, which is roughly comparable to the total thermal energy of the crystallizing white dwarf at this late phase of the evolution, markedly reduces the rate of cooling at this stage and leads to a temporary still-stand along the cooling track (Van Horn, 1968). For a given chemical composition the locus of points in the Hertzsprung-Russell diagram which mark this stillstand for stars of different masses, thus defines a 'crystallizing sequence' of white dwarfs, which is more densely populated than adjacent regions of the diagram. As is evident from Equation (1), stars consisting of elements of higher atomic number will crystallize at higher central temperatures, which are reached earlier on the cooling tracks and hence at higher luminosities. It is important to note that the rather strong $Z$-dependence of the crystallizing temperature in Equation (1) results in a significant separation in luminosity between the crystallizing sequences for different elements,

Osterbrock and O'Dell (eds.), Planetary Nebulae, 425-427. (C) I.A.U. 
thus providing the possibility of discriminating observationally among white dwarfs of different compositions.

When the crystallizing sequences predicted theoretically for different assumed compositions are compared with the observations by Eggen and Greenstein (1965), it is found that the fainter of the two sequences which they observed agrees quite well with the crystallizing sequence for stars composed principally of carbon and oxygen (Van Horn, 1968). This fainter, observed sequence contains virtually all of the hydrogendeficient objects - mainly the DC stars, which have continuous spectra - that have been identified by Weidemann (1968) as being intrinsically different in atmospheric composition from the bulk of the white dwarfs. It is therefore strongly suggestive that these stars are in fact also different in internal composition from the majority of the white dwarfs and consist almost exclusively of carbon and oxygen. This conclusion, together with (1) the observation by Greenstein and Minkowski (1964) that the central stars of several old planetaries appear to be rich in $\alpha$-particle nuclei and deficient in hydrogen, (2) the fact that there appears to be a clean separation between the hydrogenrich nebular shell and the hydrogen-poor central star - as has been pointed out by Osterbrock (1966) and by Salpeter (1968) - and (3) the observation by O'Dell (1968) that many of the fainter planetary nuclei possess continuous spectra, as do the DC white dwarfs, theretore strongly suggests that the hydrogen-deficient white dwarfs are the descendants of the central stars of planetary nebulae. The statistics of these stars are also consistent with Weidemann's (1968) result, that only $5-50 \%$ of the white dwarfs can have been formed in a planetary nebula phase, since the DC stars comprise only about $14 \%$ of the total number of white dwarfs.

The situation with respect to the brighter group of white dwarfs is unfortunately less clear, however. If Weidemann is correct and there is no luminosity gap between the two sequences that Eggen and Greenstein find to be separated in absolute magnitude, then there is no evidence for a crystallizing sequence lying above the carbonoxygen sequence, and one would therefore expect these brighter stars to consist of carbon-oxygen cores with extensive, helium-rich envelopes. On the other hand, if the luminosity gap is real, then the evidence suggests that the brighter group of white dwarfs corresponds to a crystallizing sequence which is bracketed by the crystallizing sequences for stars composed entirely of $\mathrm{Mg}^{24}$ or of $\mathrm{Fe}^{56}$. In view of the present uncertainties in the observations, however, it does not seem to be possible at present to draw any satisfactory conclusions about the composition of these stars.

Finally, it must be noted that even if the composition identification suggested for the fainter group of white dwarfs is correct, this does not constitute a proof that these stars actually are the descendants of the planetaries. On the other hand, such an hypothesis does seem to be consistent both with the observational evidence and with the inferences which can be drawn from evolutionary model calculations (Salpeter, 1968) and thus seems to indicate an evolutionary connection between the planetary nuclei and the (apparently carbon-oxygen-rich) DC white dwarfs. 


\section{References}

Eggen, O.J., Greenstein, J.L. (1965) Astrophys. J., 141, 83.

Greenstein, J.L., Minkowski, R. (1964) Astrophys. J., 140, 1601.

Kirzhnits, D. A. (1960) Soviet Physics - JETP, 11, 365.

Mestel, L., Ruderman, M.A. (1967) Mon. Not. R. astr. Soc., 136, 27.

O'Dell, C.R. (1968) in the present volume, p. 361.

Osterbrock, D.E. (1966) in Stellar Evolution, Ed. by R.F. Stein and A.G.W. Cameron, Plenum Press, New York, p. 381.

Salpeter, E.E. (1961) Astrophys. J., 134, 669.

Salpeter, E.E. (1968) in the present volume, p. 409.

Van Horn, H.M. (1968) Astrophys. J., 151, 227.

Weidemann, V. (1968) in the present volume, p. 423. 